\title{
Selective Angiography to Detect Anterior Spinal Artery Stenosis in Thoracic Ossification of the Posterior Longitudinal Ligament
}

\author{
Go Yoshida ${ }^{1}$, Hiroki Ushirozako ${ }^{1}$, Tomohiko Hasegawa ${ }^{1}$, Yu Yamato ${ }^{1}$, Tatsuya Yasuda ${ }^{1}$, \\ Tomohiro Banno ${ }^{1}$, Hideyuki Arima ${ }^{1}$, Shin Oe ${ }^{1}$, Yuki Mihara ${ }^{1}$, Tomohiro Yamada ${ }^{1}$, \\ Koichiro Ide ${ }^{1}$, Yuh Watanabe ${ }^{1}$, Takasuke Ushio ${ }^{2}$, Yukihiro Matsuyama ${ }^{1}$ \\ ${ }^{1}$ Department of Orthopedic Surgery, Hamamatsu University School of Medicine, Hamamatsu, Japan \\ ${ }^{2}$ Department of Radiology, Hamamatsu University School of Medicine, Hamamatsu, Japan
}

\begin{abstract}
Study Design: Single-center prospective study.
Purpose: To investigate anterior spinal artery (ASA) status using preoperative selective angiography in patients undergoing surgery for thoracic ossification of the posterior longitudinal ligament (T-OPLL).

Overview of Literature: Surgery for T-OPLL has a high risk of neurological complications, which might be associated with insufficient spinal cord blood flow.

Methods: This study prospectively examined nine T-OPLL patients who underwent posterior thoracic decompression with kyphosis correction and instrumented fusion at Hamamatsu University School of Medicine between 2017 and 2019. All underwent preoperative selective angiography to detect and evaluate the Adamkiewicz artery and ASA. Intraoperative neuromonitoring and Doppler ultrasonography were performed to analyze neurological complications and spinal cord blood flow.

Results: All nine patients showed ASA stenosis in the area of T-OPLL. In all patients, the Adamkiewicz artery was located between $\mathrm{T7}$ and $\mathrm{L} 2$ and the area of ASA stenosis corresponded to the level of T-OPLL and greatest spinal cord compression; intraoperative Doppler ultrasonography confirmed the ASA defect at the same spinal level. The number of spinal levels from the Adamkiewicz artery to the most compressive OPLL lesion was greater in the two patients who developed postoperative neurological deficit compared to those who did not (5.5 vs. 2.3, $p=0.014$ ).

Conclusions: This is the first study to report detection of ASA stenosis in patients with T-OPLL. Maintaining spinal cord blood flow is important in these patients to avoid neurological deterioration.
\end{abstract}

Keywords: Anterior spinal artery; Adamkiewicz artery; Ossification of the posterior longitudinal ligament; Angiography; Doppler ultrasonography

Received Nov 16, 2020; Revised Jan 25, 2021; Accepted Jan 26, 2021

Corresponding author: Go Yoshida

Department of Orthopedic Surgery, Hamamatsu University School of Medicine, 1-20-1 Handayama, Higashi-ku, Hamamatsu City, Shizuoka, 431-3192, Japan

Tel: +81-534352299, Fax: +81-534352296, E-mail: goy@k6.dion.ne.jp 


\section{Introduction}

Thoracic ossification of the posterior longitudinal ligament (T-OPLL) surgery is complicated and technically demanding because of its pathological features [1-4]. Physiological kyphosis and poor vascular supply of the spinal cord are unique characteristics of the thoracic spine that may be associated with poor surgical outcome [5]. Posterior decompression alone may be insufficient because of thoracic kyphosis; however, anterior procedures are technically demanding because of dural adhesion to the ossified ligament and the small surgical field. Neurological complications in either approach are relatively high and associated with increased rates of morbidity and mortality as well as higher healthcare costs. The reported neurological complication rate for T-OPLL surgery was $\leq 28.9 \%$ in a 2011 nationwide retrospective multicenter survey [2] and $\leq 32.2 \%$ in a 2018 nationwide prospective multicenter survey [6]. The most frequent cause of neural injury in TOPLL has been reported to be posterior decompression, although cervical or lumbar decompression was usually helpful and had a good effect on neurological status $[7,8]$. This study aimed to examine vascular and neurological status in patients undergoing T-OPLL surgery using preoperative selective angiography and intraoperative Doppler ultrasonography and neuromonitoring.

\section{Materials and Methods}

This study examined nine T-OPLL patients who underwent posterior thoracic decompression with kyphosis correction and instrumented fusion at Hamamatsu University School of Medicine between 2017 and 2019. Intraoperative transcranial motor-evoked potentials (TcMEPs), somatosensory-evoked potentials, and spinal cord-evoked potentials ( $D$-wave) were utilized in all patients. The surgical procedure involved the following steps: (1) intubation in the supine position, (2) change to prone position, (3) incision and exposure, (4) placement of bilateral pedicle screws, (5) temporary rod placement on one side, (6) posterior decompression (laminectomies), (7) kyphosis correction and fusion, and (8) closure. TcMEPs were checked after each step. In accordance with a previously reported prospective multicenter study, a $70 \%$ drop in Tc-MEP amplitude was selected as the alarm criterion to interrupt surgery using amplitudes recorded prior to the critical stage of the operation as the refer- ence baseline [9]. Follow-up in all patients was at least 1 year. Institutional review board of Hamamatsu University School of Medicine approval was obtained (approval code no., 19-229). All patients provided written informed consent.

Total intravenous anesthesia consisted of administration of propofol $(3-4 \mu \mathrm{g} / \mathrm{mL})$, fentanyl $(2 \mu \mathrm{g} / \mathrm{kg})$, and vecuronium $(0.12-0.16 \mathrm{mg} / \mathrm{kg}$ ) for induction followed by maintenance infusion of propofol $(100-150 \mu \mathrm{g} / \mathrm{kg} / \mathrm{min}$; target-controlled infusion technique), remifentanil ( $1 \mu \mathrm{g} /$ $\mathrm{kg} / \mathrm{hr}$ ), and vecuronium $(0-0.04 \mathrm{mg} / \mathrm{kg} / \mathrm{hr})$. Systolic blood pressure was maintained $>90 \mathrm{~mm} \mathrm{Hg}$.

Selective three-dimensional digital subtraction angiography (DSA) using the Alphenix (Canon, Tokyo, Japan) or Allura Xper FD 20/20 systems (Philips, Amsterdam, the Netherlands) was performed by radiologists $\leq 2$ weeks
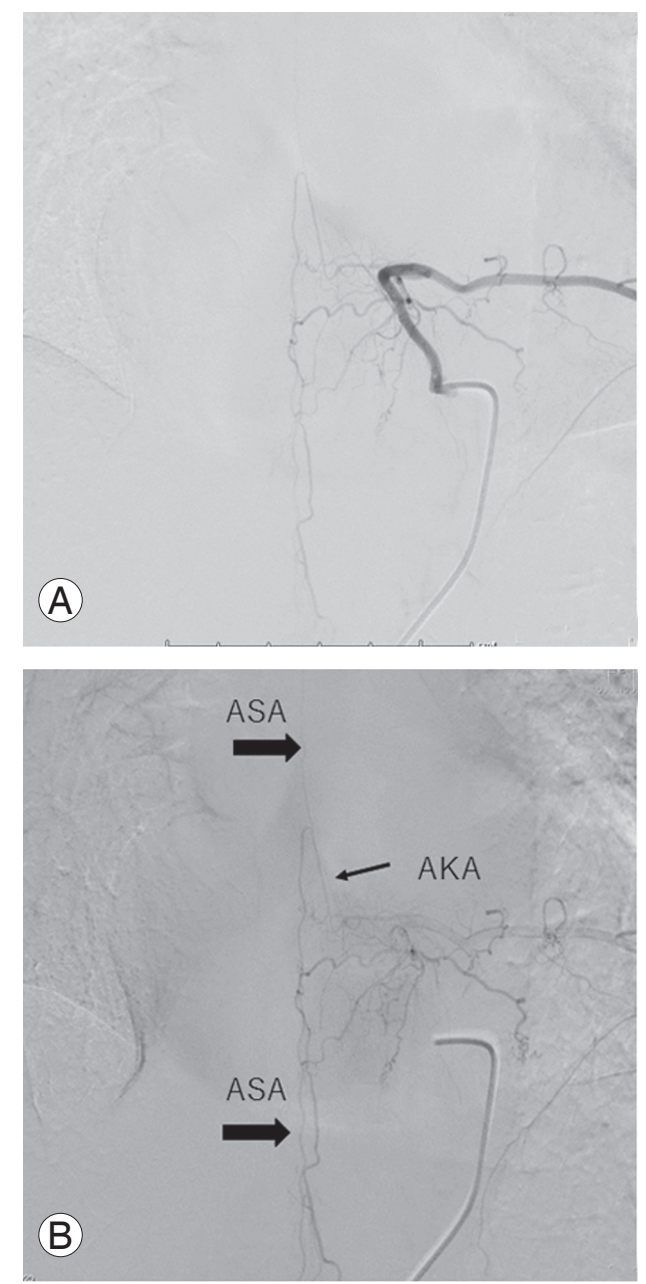

Fig. 1. Normal angiography with indications of vascular landmarks. Selective angiography from left 9th intercostal artery (A). Adamkiewicz artery (AKA) flow in ascending and descending anterior spinal artery (ASA) (B, arrows). 
before surgery to detect and evaluate the Adamkiewicz artery (arteria radicularis magna) and anterior spinal artery (ASA). DSA was selected rather than magnetic resonance angiography (MRA) because it can evaluate the ASA in relation to the ossified ligament and spinal cord movement in relation to arterial pulsations. DSA findings are shown in Fig. 1. The radiologists selectively catheterized as many intercostal or segmental arteries arising from the thoracoabdominal aorta as possible. If a radiculomedullary artery was detected, DSA was performed.

ASA continuity, Adamkiewicz artery location, and radiculomedullary arterial distribution were examined and recorded. If there was interruption of the ASA, the type (stenosis or occlusion) and spinal level of discontinuities were noted. After posterior decompression, intraoperative Doppler ultrasonography was performed to evaluate spinal cord status (floating from the OPLL or not) and ASA flow. The number of spinal levels from the Adamkiewicz artery to the most compressive OPLL lesion was counted and compared between patients with and without postoperative neurological deficit using the Student $t$-test. A $p<0.05$ was considered significant.

\section{Results}

\section{Patient characteristics and operative details}

A total of nine patients (three males and six females; mean age, 53.3 years; range, 35-68 years) were included in this study. Mean body mass index was $28.5 \mathrm{~kg} / \mathrm{m}^{2}$. Mean follow-up was 2.2 years. All patients had symptoms and signs of myelopathy in the lower extremities. Mean opera- tive time and estimated blood loss were 259 minutes and $622.8 \mathrm{~mL}$, respectively.

\section{Neurological status and motor-evoked potential monitoring}

Eight of the nine patients exhibited motor weakness before surgery (manual muscle testing [MMT] grade 3 to 5-; Frankel grade D). MEP monitoring predicted early postoperative motor deficits in two patients $(22.2 \%$, truepositive cases) and no postoperative motor deficits in seven patients (six true negative and one false positive). There was no false negative result. One patient with postoperative motor deficits experienced complete motor recovery within 6 months (case 5) and was able to walk without assistance. The other patient with deficit experienced postoperative motor weakness that persisted throughout the follow-up period (case 1).

\section{Selective angiography}

Selective angiography data are summarized in Tables 1 and 2. No angiography complications occurred. The Adamkiewicz artery was detected between T7 and L2 in all nine patients and between $\mathrm{T} 9$ and $\mathrm{T} 12$ in seven patients (77.8\%); the artery was on the left in six patients (66.7\%). In all nine patients, the ASA was visualized on the anterior surface of the spinal cord ascending from the Adamkiewicz artery and became stenotic at the level of the T-OPLL, particularly in the area of greatest spinal cord compression. The ASA above the stenosis was not visualized by selective angiography from the upper to middle

Table 1. Summary of clinical data and neurological status

\begin{tabular}{|c|c|c|c|c|c|c|c|c|c|}
\hline Case & Age/sex & Main lesion & $\begin{array}{c}\text { Occupied ratio } \\
\text { by CT }(\%)\end{array}$ & Fusion level & $\begin{array}{c}\text { Decompression } \\
\text { level }\end{array}$ & Preop MMT & Postop MMT & Preop Frankel & Postop Frankel \\
\hline 1 & $47 / F$ & T5-6 & 88 & T1-12 & T2-8, T10-12 & 3 & 1 & D & C \\
\hline 2 & $48 / F$ & T6-8 & 51 & $\mathrm{~T} 2-\mathrm{L} 1$ & T5-8, T10-11 & $5-$ & $5-$ & D & D \\
\hline 3 & $62 / \mathrm{M}$ & T6-8 & 53 & T5-T11 & T6-10 & 4 & 4 & D & D \\
\hline 4 & $58 / F$ & T5-9 & 61 & T3-T11 & T5-9 & $5-$ & $5-$ & D & D \\
\hline 5 & $51 / F$ & T3-5 & 52 & T1-T9 & $\mathrm{T} 1-7$ & $5-$ & 3 & $D$ & C \\
\hline 6 & $35 / \mathrm{M}$ & T9-10 & 51 & T1-T11 & $\mathrm{T} 2-4$ & 5 & 5 & $E$ & $E$ \\
\hline 7 & $68 / \mathrm{M}$ & T6-7 & 58 & T1-T11 & T2-8 & $5-$ & $5-$ & $D$ & $D$ \\
\hline 8 & $47 / F$ & T8-9 & 66 & T4-T12 & T7-10 & $5-$ & $5-$ & D & D \\
\hline 9 & $64 / F$ & T7-8 & 61 & T2-L1 & T6-9 & $5-$ & $5-$ & $D$ & D \\
\hline
\end{tabular}

CT, computed tomography; Preop, preoperative; Postop, postoperative; MMT, manual muscle test (lower extremities); F, female; M, male. 
Table 2. Summary of selective angiography and Doppler ultrasonography findings

\begin{tabular}{|c|c|c|c|c|c|c|c|c|}
\hline Case & $\begin{array}{l}\text { Location } \\
\text { of AKA }\end{array}$ & $\begin{array}{l}\text { Main } \\
\text { lesion }\end{array}$ & $\begin{array}{l}\text { The vertebral } \\
\text { distance }^{\text {a }}\end{array}$ & $\begin{array}{c}\text { ASA } \\
\text { stenosis }\end{array}$ & $\begin{array}{l}\text { ASA stenotic status } \\
\text { and level }\end{array}$ & $\begin{array}{l}\text { Doppler ultrasonography } \\
\text { status and level }\end{array}$ & $\begin{array}{l}\text { Neuromonitoring alarm } \\
\text { at the end of the surgery }\end{array}$ & Postop ND \\
\hline 1 & Rt. T12 & T5-6 & 6 & + & Disruption above T10 & Missing ASA above T10 & + & + \\
\hline 2 & Lt. T12 & T6-8 & 5 & + & Deficit at T6-8 & Missing ASA at T6-8 & + & - \\
\hline 3 & Rt. L2 & T6-9 & 5 & + & Disruption above T11 & Missing ASA above T11 & - & - \\
\hline 4 & Lt. T7 & T5-9 & 0 & + & Disruption above T6 & Missing ASA above T6 & - & - \\
\hline 5 & Lt. T10 & Т3-5 & 5 & + & Disruption above T5 & Missing ASA above T5 & + & + \\
\hline 6 & Lt. T11 & T9-10 & 1 & + & Disruption above T9 & Missing ASA above T9 & - & - \\
\hline 7 & Lt. Tg & T6-7 & 2 & + & Deficit at T6-7 & Not measured & - & - \\
\hline 8 & Lt. T11 & T8-9 & 2 & + & Deficit at T8-9 & Missing ASA at T8-9 & - & - \\
\hline 9 & Rt. T9 & T7-8 & 1 & + & Disruption above T8 & Missing ASA above T8 & - & - \\
\hline
\end{tabular}

AKA, Adamkiewicz artery; ASA, anterior spinal artery; Postop, postoperative; ND, neurological deficit; Rt, right; Lt, left.

${ }^{a}$ The vertebral distance between AKA and the main compressive lesion.
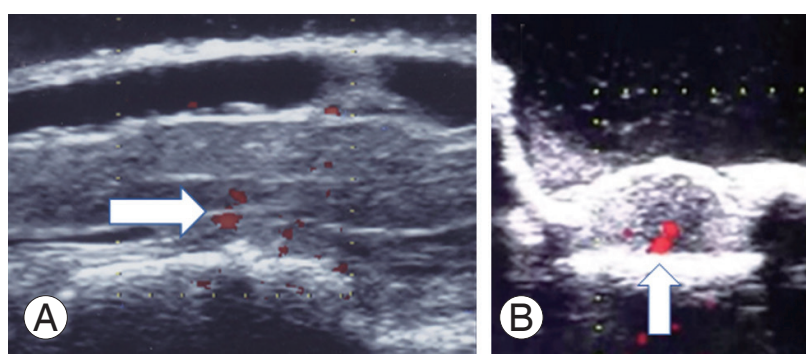

Fig. 2. Ultrasonography findings in thoracic ossification of the posterior longitudinal ligament (OPLL). (A) Sagittal Doppler image showed the compression of OPLL to spinal cord and anterior spinal artery (white arrow). (B) Axial Doppler image showed the flow of the anterior spinal artery (white arrow).

Table 3. Ultrasonography finding and postoperative motor palsy

\begin{tabular}{lcc} 
Type of cord compression & No. of patients & $\begin{array}{c}\text { Postoperative motor } \\
\text { palsy (\%) }\end{array}$ \\
\hline Floating & 1 & 0 \\
Still contact & 5 & 0 \\
Still compression & 3 & $2(66.7)$ \\
\hline
\end{tabular}

intercostal artery. The number of spinal levels from the Adamkiewicz artery to the most compressive OPLL lesion was greater in the two patients who developed postoperative neurological deficit compared to those who did not (5.5 versus $2.3, p=0.014$ ) (Table 2).

\section{Intraoperative ultrasonography}

Spinal cord status on ultrasonography after decompression
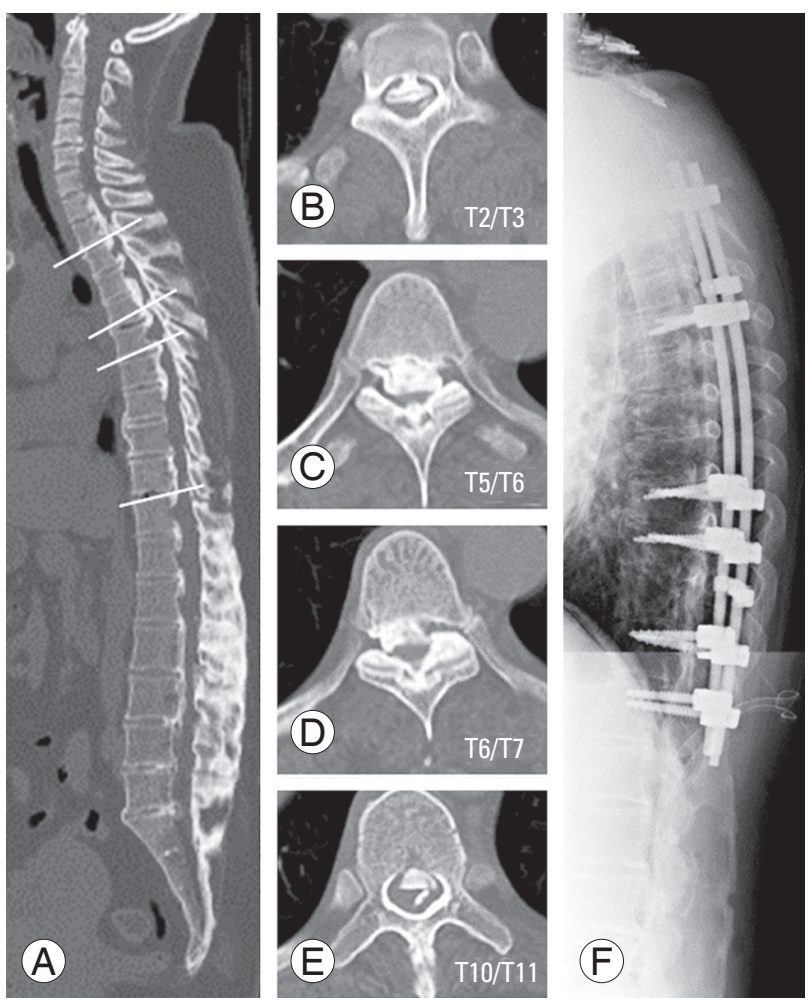

Fig. 3. Pre- and postoperative radiography in a thoracic ossification of the posterior longitudinal ligament (case 1). Sagittal view of computed tomography (A), axial views T2/3 (B), T5/6 (C), T6/7 (D), and T10/11 (E) showed severe ossification of posterior ligaments. Posterior decompression from T2 to T12 and de-kyphotic fusion from T1 to T12 were performed (F).

was floating in one patient, still in contact in five, and still compressed in three. Two of the three patients that still showed spinal cord compression experienced postoperative motor deficit (Table 3). Doppler ultrasonography was 

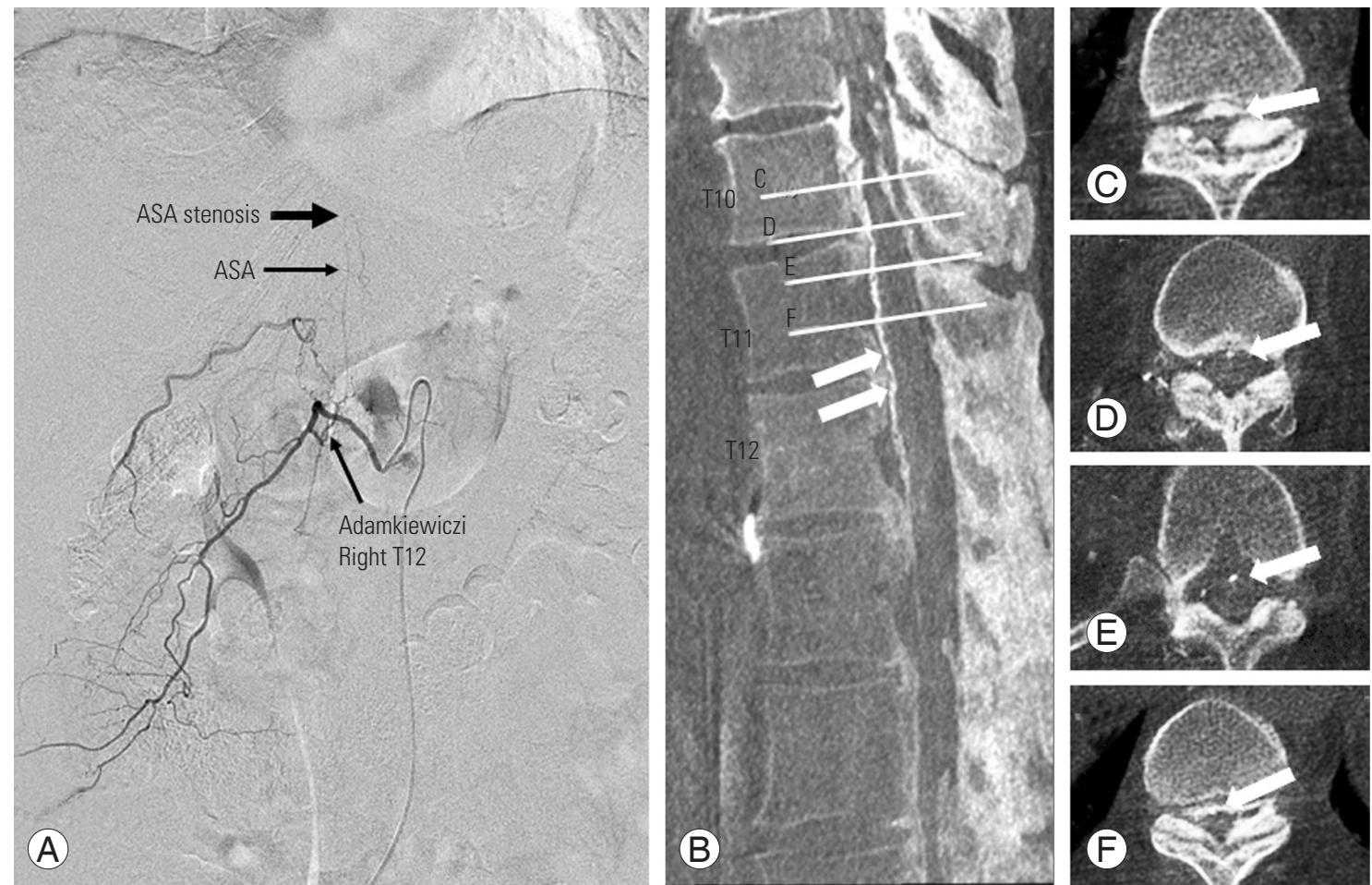

Fig. 4. Preoperative selective angiography and cone beam computed tomography (CT). (A) Digital subtraction angiography showed the anterior spinal artery (ASA) ascended from the right T12 Adamkiewicz artery; however, the ASA was occluded at the T10/T11. (B) Sagittal cone beam CT clearly showed the ASA was ascending T12 to T10. (D-F) Axial cone beam CT showed the opening of ASA from T12 to T11. (C) However, the ASA was occluded at T10/T11. White arrows indicated the ASA.

able to detect the ASA caudal to the Adamkiewicz artery but not in the areas of angiographic occlusion (Fig. 2).

\section{Case presentations}

\section{1) Case 1}

A 47-year-old female presented with severe bilateral leg weakness (MMT grade 3) and spasticity; she was unable to walk unassisted and used a wheelchair. Computed tomography showed T-OPLL from T2 to T10. Magnetic resonance imaging (MRI) showed the greatest spinal cord compression at T5/6, T2/3, and T10/11. She was treated with posterior decompression from T2 to T12 with kyphosis correction and fusion from T1 to T12 (Fig. 3). The ASA ascended from the right T12 Adamkiewicz artery on preoperative DSA and became stenotic at T10/11 (Fig. 4). Intraoperatively, MEPs deteriorated at the time of T2/T3 decompression and she developed postoperative motor deficit (MMT grade 1), which was still present 1 year after surgery. Eventually, she became able to stand with the use of bilateral short-leg orthoses.
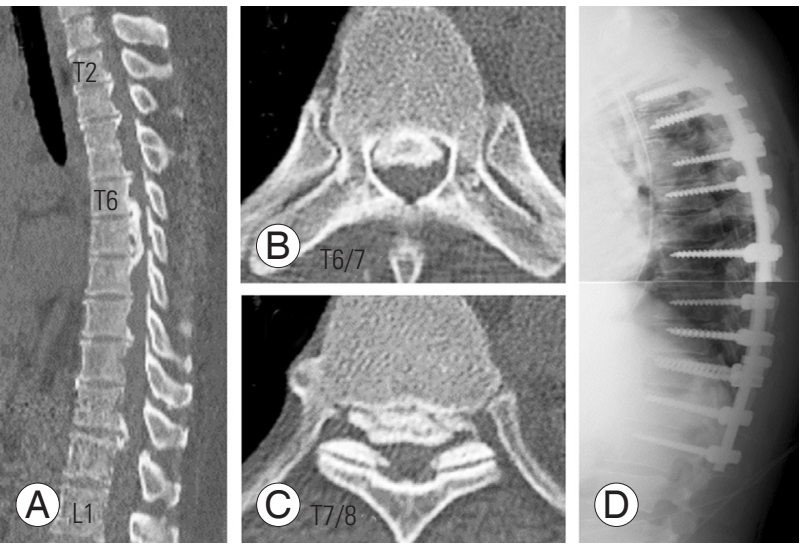

Fig. 5. Pre- and postoperative images in a thoracic ossification of the posterior longitudinal ligament (OPLL) (case 2). (A) Sagittal view showed the most compressed lesion was T6-T8. Axial view showed the T6/7 (B) and T7/8 (C) OPLL. (D) This patient was treated with posterior decompression and de-kyphotic fusion.

\section{2) Case 2}

A 48-year-old female presented with mild bilateral leg weakness (MMT grade 5-). Computed tomography showed severe T-OPLL from T6 to T8. She was treated with posterior decompression from T5 to T11 with ky- 

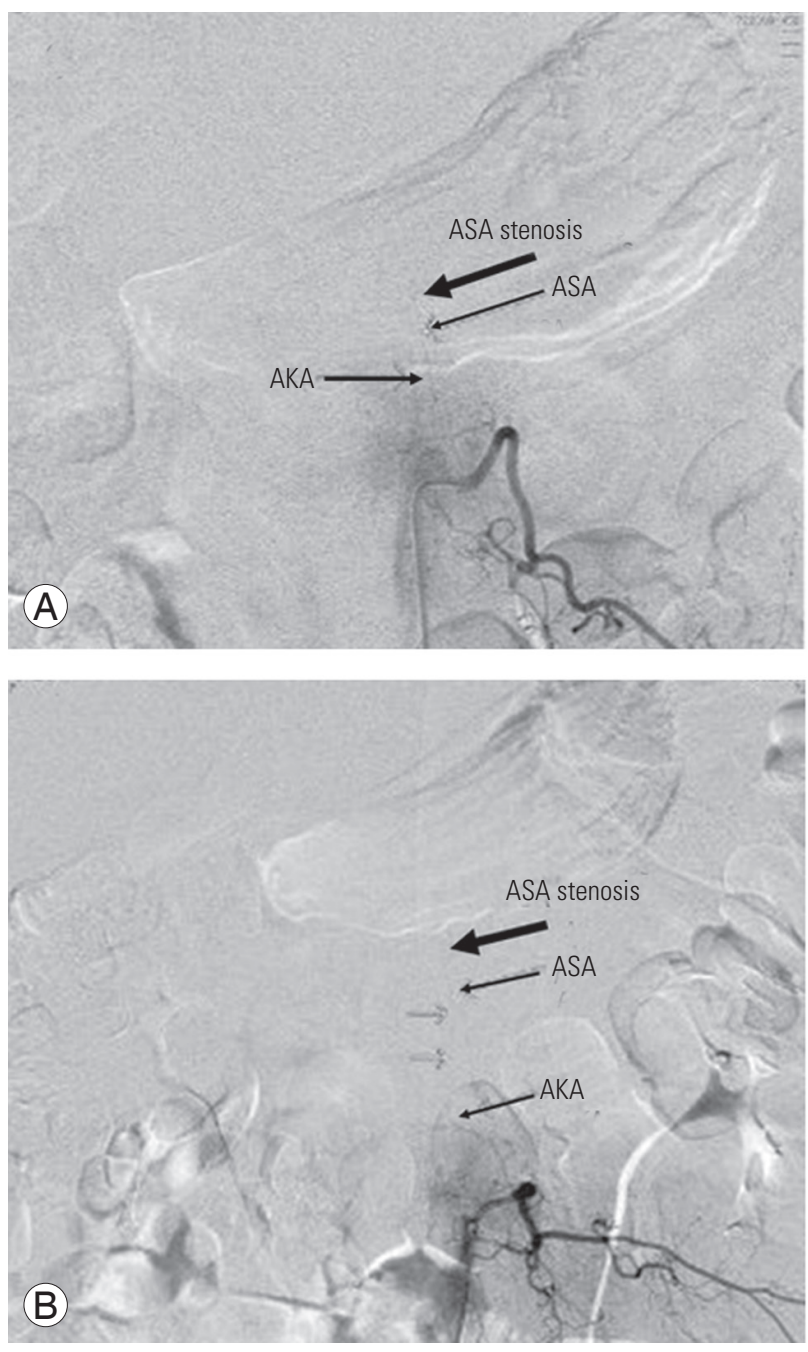

Fig. 6. (A, B) Preoperative angiography showed that the anterior spinal artery (ASA) had stenotic change at the T6 to T8 levels (arrow). AKA, Adamkiewicz artery.

phosis correction and fusion from T2 to L1 (Fig. 5). The ASA ascended from the left T12 Adamkiewicz artery on preoperative DSA and became stenotic from T6 to T8 (Fig. 6). Intraoperatively, MEPs deteriorated at the time of T6/ T7 decompression but partially recovered at the end of surgery. She had no postoperative neurological deficit.

\section{Discussion}

T-OPLL surgery has a higher complication rate than cervical OPLL surgery. In a previous multicenter study, approximately $30 \%$ of the patients with T-OPLL had a neurological deficit caused by posterior decompression with kyphosis correction and fusion [2,6,7]; the most frequent timing of intraoperative MEP deterioration occurred during decompression $[7,8]$. The reason decompression is related to this deterioration is unclear, but possibilities include iatrogenic injury, alignment change, and spinal cord ischemia. During posterior decompression, vibration or heat from the high-speed burr might cause spinal cord injury. In addition, adhesions between the dura and ossified ligament can result in tearing of the dura during decompression. Moreover, posterior decompression might accentuate thoracic kyphosis due to removal of posterior elements.

Regarding spinal cord ischemia, cord infarction is difficult to visualize on diagnostic imaging. Although diffusion-weighted MRI can demonstrate cord ischemia, it is difficult to clarify the relationship between the ischemic area and OPLL lesion. For diagnostic cerebral imaging, noninvasive vascular imaging modalities, such as computed tomography angiography and MRA, have replaced DSA for routine imaging indications [10]. Although approximately $80 \%$ of Adamkiewicz arteries are detectable on MRA [11,12], selective angiography is the gold standard for identifying, localizing, and classifying status of spinal arteries. Furthermore, DSA remains the preferred choice to study detailed angiographic architecture because of spinal cord movement and the small diameter of spinal arteries. The ASA supplies the anterior horn and anterior portions of the lateral column on the left or right side at each spinal cord level. If surgical manipulation affects ASA blood flow, spinal cord ischemia might occur because of pre-existing ASA stenosis due to ligament ossification. According to a cadaver study, normal ASA diameter ranges from 468 to $1,120 \mu \mathrm{m}$; the artery courses ventromedially along the spinal cord without interruption at thoracic spinal levels [13]. ASA continuity is controversial. Biglioli et al. [14] and Svensson [15] independently reported that the ASA is continuous from the vertebral arteries to the filum terminalis. However, Gharagozloo et al. [16] reported that the ASA is discontinuous and the blood supply of the spinal cord is segmental via vertebral, intercostal, and lumbar arteries. If the ASA is continuous on the ventral side of the spinal cord, the thoracic spinal cord might be supplied from distant sources, such as the vertebral or subclavicular arteries, even if the Adamkiewicz or anterior radiculomedullary arteries are blocked or occluded. However, the ASA is stenotic in T-OPLL, so posterior decompression resulting in anterior radiculomedullary artery occlusion via nerve root injury or sacrifice might cause spinal cord infarction. Generally, laminec- 
tomy reduces spinal blood flow by approximately $22 \%$ to $40 \%$ [17-19]. This decrease may cause new or worse motor deficit in a spinal cord with tenuous blood supply.

Impaired ASA blood flow in T-OPLL has three possible causes. One is direct ASA compression by ossified ligament. Hashizume et al. [20] reported compression of the spinal cord in a study of pathological findings in cervical OPLL patients; the cord was flattened by the OPLL and necrosis was observed within gray matter of the cord [21]. Zhang and Wang $[22,23]$ performed computed tomography angiography in patients with cervical spondylotic myelopathy (CSM) and found that $20 \%$ had mild ASA occlusion. In both CSM and cervical OPLL, ASA occlusion might be rare because there is space between the PLL and spinal cord due to cervical lordosis. However, in T-OPLL, there is no space because of thoracic kyphosis and ossified ligament. Another possible cause is anterior radiculomedullary artery occlusion. Some patients have a horizontally wide OPLL that compresses the anterior radiculomedullary artery on the lateral edge of the spinal cord. In our study, selective angiography of each anterior radiculomedullary artery was not performed because of the risk of thrombosis, but some of those vessels were not detected because they were occluded. We observed collateral blood flow from small perivertebral or pial arteries in three of our nine patients. Perivertebral anastomoses or blood supply from upper thoracic radiculomedullary arteries might maintain blood flow when the ASA is compromised in TOPLL. Gailloud [24] emphasized the importance of the upper thoracic anterior radiculomedullary artery, which supplies the ASA. Finally, intravascular pathology, such as endothelial hyperplasia or lipid accumulation, can also impair ASA blood flow; however, this has not been examined in T-OPLL patients or cadavers. Regardless of reason, the ASA in our T-OPLL frequently showed signs of stenosis. Therefore, constant spinal cord blood flow should be preserved before and after the decompression maneuver.

Prostaglandin E1 (PGE1) might improve spinal cord blood flow in T-OPLL decompression surgery while maintaining adequate blood pressure. PGE1 is the cyclooxygenase metabolite of dihomo- $\gamma$-linolenic acid and its actions include vasodilation, hypotension, and anti-platelet effects. A previous study showed that PGE1 maintains ASA and anterior spinal cord blood flow in cervical posterior decompression surgery [25]. In cardiovascular surgery, particularly aortic dissection procedures, it is used intraoperatively to prevent anterior spinal cord syndrome, a frequent complication of aortic replacement surgery [26,27]. Studies of PGE1 in patients undergoing T-OPLL surgery are warranted.

Our study has several limitations. First, we included only recent patients with severe T-OPLL who underwent surgery; those with mild OPLL or who received conservative treatment did not undergo angiography because of its invasiveness and risk. Second, we did not include patients who underwent anterior decompression, which might have affected our results. Third, we only analyzed T-OPLL, although OPLL was usually observed in the cervical and lumbar spine as well. The effect of multiple lesions should be evaluated in future angiographic studies. Finally, we did not investigate the effects of medications.

\section{Conclusions}

In conclusion, ASA occlusion was observed on selective spinal angiography in all patients with severe T-OPLL. Treatment strategies should consider spinal cord blood supply status. Medications that improve spinal cord blood flow might be effective to prevent postoperative neurological deficits in this patient population.

\section{Conflict of Interest}

Dr. Oe and Yamato belong to a donation-funded laboratory called the "Division of Geriatric Musculoskeletal Health." Donations to this laboratory have been received from Medtronic Sofamor Danek Inc., Japan Medical Dynamic Marketing Inc., and the Meitoku Medical Institution Jyuzen Memorial Hospital. No potential conflict of interest relevant to this article was reported.

\section{Author Contributions}

Dr. Yoshida was responsible for the study's conception and design. Dr. Yoshida acquired, analyzed, and interpreted data; drafted the article; and approved the final version on behalf of all authors. All authors critically revised the article and reviewed the submitted version. Dr. Matsuyama supervised the study.

\section{References}

1. Matsuyama Y, Yoshihara H, Tsuji T, et al. Surgical outcome of ossification of the posterior longitudinal 
ligament (OPLL) of the thoracic spine: implication of the type of ossification and surgical options. J Spinal Disord Tech 2005;18:492-7.

2. Matsumoto M, Toyama Y, Chikuda H, et al. Outcomes of fusion surgery for ossification of the posterior longitudinal ligament of the thoracic spine: a multicenter retrospective survey: clinical article. J Neurosurg Spine 2011;15:380-5.

3. Matsunaga S, Nakamura K, Seichi A, et al. Radiographic predictors for the development of myelopathy in patients with ossification of the posterior longitudinal ligament: a multicenter cohort study. Spine (Phila Pa 1976) 2008;33:2648-50.

4. Kawahara N, Tomita K, Murakami H, et al. Circumspinal decompression with dekyphosis stabilization for thoracic myelopathy due to ossification of the posterior longitudinal ligament. Spine (Phila $\mathrm{Pa}$ 1976) 2008;33:39-46.

5. Lazorthes G, Gouaze A, Zadeh JO, Santini JJ, Lazorthes Y, Burdin P. Arterial vascularization of the spinal cord: recent studies of the anastomotic substitution pathways. J Neurosurg 1971;35:253-62.

6. Imagama S, Ando K, Takeuchi K, et al. Perioperative complications after surgery for thoracic ossification of posterior longitudinal ligament: a nationwide multicenter prospective study. Spine (Phila Pa 1976) 2018;43:E1389-97.

7. Yoshida G, Ando M, Imagama S, et al. Alert timing and corresponding intervention with intraoperative spinal cord monitoring for high-risk spinal surgery. Spine (Phila Pa 1976) 2019;44:E470-9.

8. Ando K, Kobayashi K, Machino M, et al. Wave changes in intraoperative transcranial motor-evoked potentials during posterior decompression and dekyphotic corrective fusion with instrumentation for thoracic ossification of the posterior longitudinal ligament. Eur J Orthop Surg Traumatol 2019;29:117785.

9. Kobayashi S, Matsuyama Y, Shinomiya K, et al. A new alarm point of transcranial electrical stimulation motor evoked potentials for intraoperative spinal cord monitoring: a prospective multicenter study from the Spinal Cord Monitoring Working Group of the Japanese Society for Spine Surgery and Related Research. J Neurosurg Spine 2014;20:102-7.

10. Chappell ET, Moure FC, Good MC. Comparison of computed tomographic angiography with digital sub- traction angiography in the diagnosis of cerebral aneurysms: a meta-analysis. Neurosurgery 2003;52:62431.

11. Hyodoh H, Shirase R, Kawaharada N, et al. MR angiography for detecting the artery of Adamkiewicz and its branching level from the aorta. Magn Reson Med Sci 2009;8:159-64.

12. Taterra D, Skinningsrud B, Pekala PA, et al. Artery of Adamkiewicz: a meta-analysis of anatomical characteristics. Neuroradiology 2019;61:869-80.

13. Biglioli P, Spirito R, Roberto M, et al. The anterior spinal artery: the main arterial supply of the human spinal cord: a preliminary anatomic study. J Thorac Cardiovasc Surg 2000;119:376-9.

14. Biglioli P, Roberto M, Cannata A, et al. Upper and lower spinal cord blood supply: the continuity of the anterior spinal artery and the relevance of the lumbar arteries. J Thorac Cardiovasc Surg 2004;127:1188-92.

15. Svensson LG. Management of segmental intercostal and lumbar arteries during descending and thoracoabdominal aneurysm repairs. Semin Thorac Cardiovasc Surg 1998;10:45-9.

16. Gharagozloo F, Larson J, Dausmann MJ, Neville RF Jr, Gomes MN. Spinal cord protection during surgical procedures on the descending thoracic and thoracoabdominal aorta: review of current techniques. Chest 1996;109:799-809.

17. Anderson DK, Means ED. The effect of laminectomy on spinal cord blood flow, energy metabolism and ATPase activity. Paraplegia 1985;23:58.

18. Anderson DK, Nicolosi GR, Means ED, Hartley LE. Effects of laminectomy on spinal cord blood flow. J Neurosurg 1978;48:232-8.

19. Kobrine AI, Doyle TF, Martins AN. Spinal cord blood flow in the rhesus monkey by the hydrogen clearance method. Surg Neurol 1974;2:197-200.

20. Hashizume Y, Iijima S, Kishimoto H, Yanagi T. Pathology of spinal cord lesions caused by ossification of the posterior longitudinal ligament. Acta Neuropathol 1984;63:123-30.

21. Kameyama T, Hashizume Y, Ando T, Takahashi A, Yanagi T, Mizuno J. Spinal cord morphology and pathology in ossification of the posterior longitudinal ligament. Brain 1995;118(Pt 1):263-78.

22. Zhang Z, Wang H. CT angiography of anterior spinal artery in cervical spondylotic myelopathy. Eur Spine J 2013;22:2515-9. 
23. Zhang Z, Wang $H$. Is the "snake-eye" MRI sign correlated to anterior spinal artery occlusion on CT angiography in cervical spondylotic myelopathy and amyotrophy? Eur Spine J 2014;231541-7.

24. Gailloud P. The artery of von Haller: a constant anterior radiculomedullary artery at the upper thoracic level. Neurosurgery 2013;73:1034-43.

25. Tsuji T, Matsuyama Y, Sato K, Iwata H. Evaluation of spinal cord blood flow during prostaglandin E1induced hypotension with power Doppler ultrasonography. Spinal Cord 2001;39:31-6.
26. DePalma RG, Olding M, Yu GW, et al. Vascular interventions for impotence: lessons learned. J Vasc Surg 1995;21:576-84.

27. Ohtake H, Urayama H, Katada S, et al. Prevention of spinal cord ischemia by selective intercostal arterial infusion of prostaglandin E1. J Vasc Surg 1998;28:301-7. 